\title{
Autism mouse models cluster by brain activity pattern
}

\section{BY NIKO MCCARTY}

10 SEPTEMBER 2021

Brain scans from 16 mouse models of autism reveal at least four distinct patterns of brain activity, a new study suggests. The findings lend fresh support to the popular idea that autism is associated with a range of brain 'signatures.'

Telltale neural signatures of autism have long proved elusive, with functional magnetic resonance imaging (fMRI) and other brain scanning technologies shouldering the blame for scattered and inconsistent results.

"One big question is whether there's a single signature of dysfunction in the brain of people with autism. And many people consider that to be, like, something that there must be," says study investigator Alessandro Gozzi, senior researcher at the Istituto Italiano di Tecnologia in Rovereto, Italy. "If we've not found it yet, the blame must be on the method: fMRI." The method gauges small changes in blood flow and oxygenation as an indirect measure of brain activity.

For the new study, published in Molecular Psychiatry in August, Gozzi and his colleagues used fMRI to study brain connectivity patterns - or which brain regions 'talk' to each other, and to what degree. Brain regions are considered to be in communication if they have synchronous oscillations in blood flow.

To tackle the question of reproducibility in $\mathrm{fMRI}$, the researchers conducted their analysis in mice, anesthetizing the animals and fixing their heads in place to prevent any motion that could perturb brain signals. "We moved to a model organism where we can control, in exquisite detail, many of the factors that are considered to be at the basis of this variability, this unreliability in imaging," Gozzi says.

The 16 mouse models fall into four groups based on brain activity patterns, the analysis found. For example, one group shows increased connectivity between areas in the prefrontal cortex and 


\section{Spectrum | Autism Research News}

https://www.spectrumnews.org

amygdala, social and emotional regions of the brain, respectively; another group has increased connectivity between the hippocampus and ventral orbital cortex, regions involved in memory and higher cognitive functions.

"I think we know, from a behavioral point of view, that autism is very heterogeneous," says Kaustubh Supekar, clinical assistant professor of psychiatry and behavioral sciences at Stanford University in California, who was not involved in the study. "Expecting that we were going to find a common signature for such a heterogeneous condition was sort of a pipe dream."

\section{Clustered connections:}

Gozzi and his colleagues, at locations in Italy and Switzerland, imaged 350 animals, including controls and roughly the same number from each of the 16 autism mouse models: 13 models with autism-linked mutations, including in the genes SHANK3, FMR1 or MECP2; mice deficient in microglia, immune cells that sculpt neuronal connections; mice exposed prenatally to the immune signaling molecule IL-6; and an inbred strain called BTBR.

A machine-learning model trained on the $\mathrm{fMRI}$ data clustered those results into four groups. Mice with mutations in the genes CHD8 or SYN2, those with deletions in chromosomal region 16p11.2, and the BTBR and IL6 models all have reduced neuronal signaling between the cortex, striatum and inferior colliculus, but increased signals between the ventral orbital cortex and hippocampus.

By contrast, mice with mutations in SHANK3, CNTNAP2 and SGSH, have reduced activity between the hippocampus and insula, and a moderate increase between the nucleus accumbens and hypothalamus.

The other mouse models, such as those with mutations in CDKL5 or MECP2, or animals with missing microglia, clustered into two additional groups with entirely different neuronal activity patterns.

\section{Clinical start:}

Though it focused on mice, the new study could have clinical implications, researchers say.

"This clustering of different subgroups of animal models really provides a first step in trying to understand what could be expected in humans," says Itamar Kahn, associate professor of neuroscience at Columbia University, who was not involved in the study.

People and mice harboring a 16p11.2 deletion, for example, have similar changes in brain connectivity, Gozzi's group previously showed.

But Gozzi's use of anesthesia could be problematic, says Vinod Menon, professor of psychiatry 


\section{Spectrum | Autism Research News}

https://www.spectrumnews.org

and behavioral sciences at Stanford University, who was not involved in the study.

"The anesthetized brain is very different from an awake, behaving brain," he says. "There's no question this study will have a big impact, and I think it's an important step, but it will be interesting to see what happens in the case of non-anesthetized mice as well."

For future studies, Gozzi says he plans to triple the number of autism animal models scanned, which may yield additional brain connectivity 'clusters.'

"We want to scan at least 50 [mouse models] to begin to talk about how different mutations can be grouped based on connectivity," he says.

Scanning mice with one mutation at a time, though, may not accurately reflect autism's complexity. Many autistic people harbor numerous mutations that contribute to their condition.

"We know these genes don't work in isolation," Supekar says. "So then this becomes an exponentially complex thing, where you need to create models with a lot of genes knocked out at once."

Cite this article: https://doi.org/10.53053/JUPD6575 